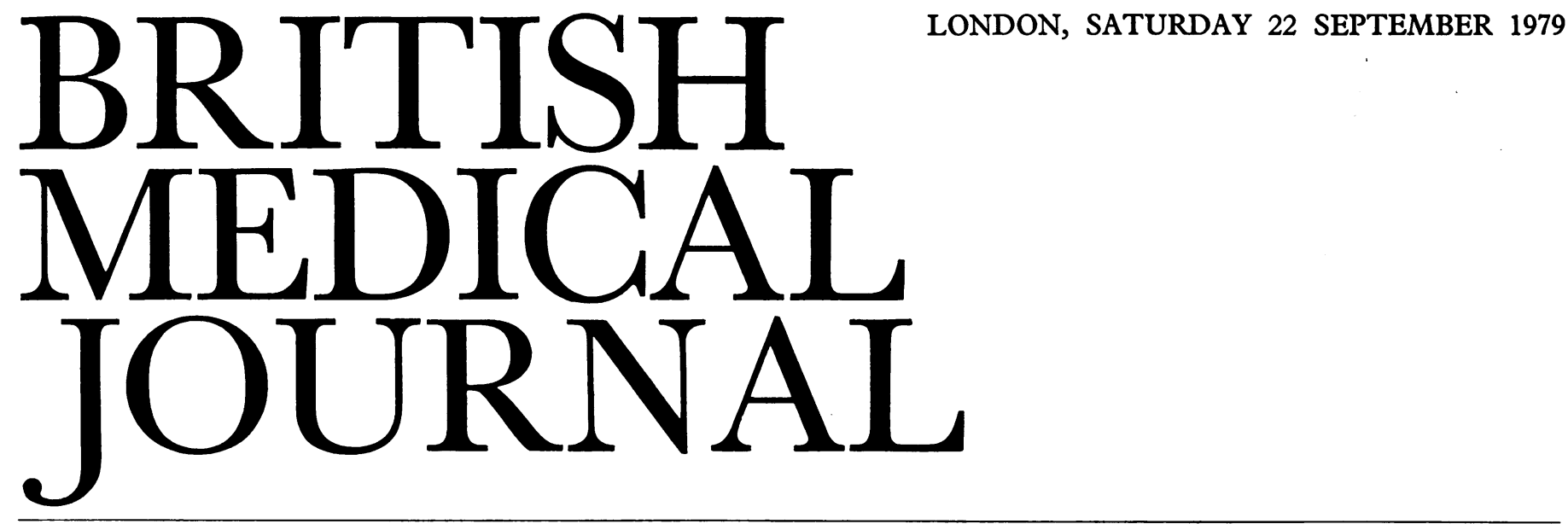

\title{
Cancer of the ovary
}

Cancer of the ovary is now the fourth most common cause of death from cancer in British women and the most common fatal gynaecological malignancy. As deaths from carcinoma of the uterine cervix have fallen in the past 20 years those from ovarian carcinoma have steadily risen from 2702 in 1958 to 3486 in 1974 . The pattern is the same in the United States, where there is a death from carcinoma of the ovary every 50 minutes.

World statistics suggest that in women of European or American origin the incidence of ovarian carcinoma is three to four times higher in Asian and African women. Since, however, in countries where the disease is relatively common (Israel, Denmark, the United States, England) the maximum incidence is in women aged 55-65 years, these racial differences may be due in part to variations in life expectancy. That would not, however, explain completely the very low incidence of the disease found, for example, in Japan and Chile. Death rates in Japanese migrants in Hawaii and their first-generation offspring are substantially higher than those in Japan. ${ }^{1}$

Ovarian carcinoma is seen most often in highly industrialised countries, which may suggest that physical or chemical carcinogens play a part-but again Japan provides a puzzling exception. Evidence associating the carcinoma with asbestos and talc, for instance, has been inconclusive, though several chemical carcinogens, including 20-methylcholanthrene and 7,12-dimethyl benz $(a)$ anthracene, have been shown to induce neoplasms in the ovaries of rodents. ${ }^{2}$ There is a slight increase over the expected frequency of carcinoma after irradiation of the pelvic organs. ${ }^{3}$ Schoenberg et $a l^{4}$ and others who have studied women with multiple primary cancers have suggested an association between the aetiological determinants of ovarian and breast cancers. In fact, women with cancer of the breast have twice the standard risk of subsequently developing a separate primary cancer of the ovary, and women with a cancer of the ovary are three to four times more likely to develop a separate primary carcinoma of the breast.

While Wynder et $a l^{5}$ found no significant differences in marital or pregnancy histories in patients with ovarian carcinoma when compared with controls, the experience of most clinicians probably accords with findings from Israel showing that such patients (like those with breast cancer) are more likely never to have married, or to have had a late pregnancy and to be relatively infertile. ${ }^{6}$ In Bombay, for example, Jussawala et $\mathrm{al}^{7}$ found that Parsi women, who tend to have those characteristics, have more cancers of breast, ovary, and endometrium, and fewer of the cervix, than do other women of that city, who tend to marry and have children while still young. A familial risk of ovarian carcinoma is also well recognised; and a higher than expected incidence of ovarian tumours, both benign and malignant, is found in women with two multisystem syndromes inherited as autosomal dominants-PeutzJegher's syndrome ${ }^{8}$ and inherited basal naevus syndrome. ${ }^{9}$

The occasional concurrence of adenocarcinomas of the endometrium and the endometrioid type of adenocarcinoma of the ovary suggests a common proliferative response of susceptible cells. Nevertheless, there is little solid evidence for any hormone dependency of ovarian cancers; certainly, the hypertension-obesity-diabetes triad associated with enhanced risk of endometrial cancer has not been observed in patients with ovarian cancer. No association between oral contraceptives and ovarian carcinoma has so far been confirmed, 10 though the action of hormonal contraception in delaying and reducing parity may have long-term effects.

Foci of endometriosis can be observed in the ovaries of many women at laparotomy and necropsy, and very occasionally carcinomas can originate from these foci. Nevertheless, they are not always of the endometrioid type, and association is unlikely to be causal. The explanation seems to be that both ovarian cancer and endometriosis occur in women who share similar demographic characteristics of relative infertility and delayed parity. Attempts to isolate viruses from cultured human ovarian cancer cells have so far been unsuccessful and ovarian neoplasms have not been induced in animal experiments by oncogenic viruses. The mumps virus ${ }^{11}$ has been exonerated as a cause, and a negative association has even been postulated. ${ }^{512} \mathrm{~A}$ possible association between ovarian carcinoma and women with group A blood has been made by Osborne and Degeorge. ${ }^{13}$

Changes in the ability to metabolise anticonvulsant drugs and interference with or potentiation of carcinogens are thought to contribute to the surprising occurrence of thecomas in the ovaries of epileptic children, though one large survey of epileptic hospital patients on long-term anticonvulsant therapy found no excess of neoplasms of any type. ${ }^{14}$

Ovarian tumours were among the first to respond to the early chemotherapeutic agents, but there is still no clearcut treatment policy. The range of drugs has been expanded recently and the exciting possibilities offered by cis-platinum diamminodichloride are especially encouraging. Such advances as have been made have been produced not by explorations of existing theories of histogenesis or aetiology but almost by chance. ${ }^{15}$ Alkylating agents such as chlorambucil, cyclo- 
phosphamide, and melphalan given individually produce a remission in a quarter to a third of patients with advanced ovarian tumours, but combinations of these drugs have been disappointing, with no increase in that proportion. The addition of cis-platinum diamminodichloride even in relatively low doses has doubled these response rates. ${ }^{16}$ Other agents, such as hexamethyl melamine, adriamycin, ifosfamide, peptichemio, and the new podophyllotoxins (including VP16), are being evaluated. Even the best chemotherapeutic combinations, however, may be ineffective without surgical treatment to reduce the bulk of the tumour.

Current management of advanced ovarian carcinoma requires the removal of as much tumour as safely possible at initial laparotomy, along with both ovaries, uterus, and omentum (in which sites occult disease may reside). In order to render any inoperable disease removable, a chemotherapeutic response should be established as soon as possible, and, after maximum response, another laparotomy should be performed to remove any residual tumour, along with multiple biopsies of suspicious areas. Further adequate chemotherapy is indicated to eradicate any residual macroscopic or microscopic disease. Greater accuracy in the choice of chemotherapy for individual patients may, in the future, be obtained by drug selection challenges on the xenografted tumours in immune deficient mice or cell colonies obtained from laparotomy specimens.

Advanced ovarian carcinoma is found in as many as $70 \%$ of all patients at presentation. The median survival is about eight months from diagnosis. Any improvement on that grim prognosis requires a combination of surgical expertise and determined chemotherapy; and compromise in either modality is likely to result in relapse and rapid death.

1 Haenszel, W, and Kurihara, M, Fournal of the National Cancer Institute, $1968,40,43$.

2 Toth, B, Cancer Research, 1970, 30, 2583.

${ }^{3}$ British Medical fournal, 1972, 2, 365.

4 Schoenberg, B S, Greenberg, R A, and Eisenberg, H, Fournal of the National Cancer Institute, 1969, 43, 15.

5 Wynder, E L, Dodo, H, and Barber, H R K, Cancer, 1969, 23, 352.

6 Sterwart, H L, et al, fournal of the National Cancer Institute, 1966, 37, 1.

7 Jussawalla, D J, et al, British Fournal of Cancer, 1970, 24, 56. 8 Christian, C D, American fournal of Obstetrics and Gynecology, 1971, 111,
529 .

${ }^{9}$ Berlin, N I, et al, Annals of Internal Medicine, 1966, 64, 403.

10 Committee on Safety of Medicines, Carcinogenicity Tests of Oral Contraceptives. London, HMSO, 1972.

${ }^{11}$ Adelstein, A M and Donovan, J W, British Medical fournal, 1972, 4, 629.

12 West, R O, Cancer, 1966, 19, 1001.

13 Osborne, R H, and Degeorge, F V, American Fournal of Human Genetics. $1963,15,380$.

14 Clemmesen, J, Fuglsang-Frederiksen, V, and Plum, C M, Lancet, 1974, 1, 705 .

15 Rosenberg, B, et al, Nature, 1969, 222, 385

16 Wiltshaw, E, and Barker, G $\mathrm{H}$, to be published.

\section{Transplant Olympics}

Last month 200 men and women between the ages of 14 and 44 assembled at Castle Field in Portsmouth to take part in the second Transplant Olympics. As athletes they shared one characteristic: each had a well-functioning kidney transplant. Their performance in the 33 separate events may not have reached Olympic or even sports clubs standards, but their times were certainly better than might be expected from, say, their doctors. The 1500 metres was won in a modest six minutes by Paul West of Liverpool-the sort of time that any ordinary young man or woman in the street might be able to accomplish with some training. Again, the tennis champions, men and women, were certainly not of Wimbledon class but they would have felt comfortable on the first ladder of their local club.

These games were not, then, for the handicapped: they were for men and women with transplant kidneys, of one to eight years' vintage, who wanted to demonstrate their normality. Competitors (six for each team) came from England, Scotland, Wales, and Northern Ireland and from countries overseas as far afield as Mexico, Israel, and Greece. Their aim was to be ambassadors for kidney transplantation and to draw attention to their normality in the hope that this would persuade the spectators to become potential kidney donors and thus help the thousands of patients on dialysis to achieve a similarly normal, independent state of existence. Over 5000 donor cards were signed at the meeting and taken away.

The organising committee and its chairman, transplant surgeon Mr Maurice Slapak of St Mary's Hospital, Portsmouth, decided that no individual records of times or distances would be kept, though gold, silver, and bronze medals were awarded to the winners of the varying events. The champion team was Eire, with its flying Irishman Maxie Scully, the winner of three gold medals in highly creditable times. The most colourful entrant was a Mexican who did not compete-he had a wooden leg-but instead he entertained the others with his guitar.

Six separate bids were entered by countries who wanted to stage next year's Olympics, including an imaginative Canadian bid for a Winter Olympics in 1981 in the Rockies. The International event was awarded to the United States and will be staged in September 1980 at Lake Placid. The Transplant Olympic Games to choose the British team will be staged in August 1980 in Birmingham.

\section{Liver dysfunction in inflammatory bowel disease}

Patients with ulcerative colitis or Crohn's disease may develop extraintestinal complications of the skin, eyes, joints, or renal tract. ${ }^{1}$ Various hepatobiliary disorders are also associated with inflammatory bowel disease, including pericholangitis, ${ }^{2}$ fatty change, chronic active hepatitis, cirrhosis, granulomas, amyloidosis, hepatic abscesses, ${ }^{3}$ gall stones, ${ }^{45}$ sclerosing cholangitis, ${ }^{6}$ and carcinoma of the biliary tract. ${ }^{78}$

Minor histological abnormalities are equally common in Crohn's disease and ulcerative colitis, but their prevalence depends on the source of the pathological material. Roughly two-fifths of surgical biopsy and postmortem specimens show fatty change, a reflection of the poor nutritional state or septicaemia of some patients during an acute attack. ${ }^{910}$ Pericholangitis, also called portal triaditis, is recognised histologically by cellular infiltration of the portal tracts, portal fibrosis, and concentric fibrosis around the bile ducts, and is found in over half of surgical biopsy specimens. ${ }^{910}$ In clinical studies most liver biopsy samples have been obtained from patients with abnormal serum enzyme concentrations or bromsulphalein retention, and in one such series fatty change and pericholangitis were each found in about $7 \%$ of patients with inflammatory bowel disease. ${ }^{11} 12$ It is important not to mistake such histological changes, which are of little clinical importance, for those of serious liver disease. Fortunately the latter is 